\title{
Bem-estar subjetivo em mães de crianças sorointerrogativas para o HIV/AIDS
}

(Subjective well-being in mothers of children with an undefined diagnosis of soropositivity for HIV/AIDS)

(Bienestar subjetivo de las madres de niños seropositivos al HIV/AIDS)

\author{
Flávio Lúcio Almeida Lima* \\ Ana Alayde Werba Saldanha* \\ Josevânia da Silva Cruz de Oliveira ${ }^{* *+}$
}

\begin{abstract}
Resumo
Este estudo teve por objetivo verificar o bem-estar subjetivo em mães que têm crianças com diagnóstico sorointerrogativo para o HIV, atendidas por um SAE (Serviço de Atendimento Especializado) na cidade de João Pessoa-PB. A amostra de conveniência foi composta por 30 mães que têm crianças com diagnóstico de soropositividade indefinido, com idades variando de 19 a 39 anos $(M=27, D P=5,18)$. Foram aplicados a escala de bem-estar subjetivo (EBES) e um questionário sociodemográfico. Os dados foram processados pelo programa estatístico SPSS (Statistical Package for the Social Sciences) e analisados por meio de estatística bivariada (teste $\mathrm{t}$ de Student; análise de variância). Os resultados indicaram instabilidade no bem-estar subjetivo e associação do fator "afetos positivos" com o "tempo de cuidado". Concluiu-se que a vivência de uma situação interrogativa diante do HIV/AIDS emana sentimentos diversos que têm relação com a história da epidemia, o enfrentamento e as implicaçóes para os diversos domínios pessoais, entre eles, a maternidade.
\end{abstract}

Palavras-chave: transmissão vertical; HIV/AIDS; bem-estar subjetivo.

\footnotetext{
Texto recebido em agosto/2008 e aprovado para publicação em abril/2009.

Psicólogo e mestre em Psicologia Social pela Universidade Federal da Paraíba - UFPB, e-mail: flaviopsicopb@yahoo.com

** Doutora em Psicologia, professora adjunta, Programa de Pós-Graduaçāo em Psicologia Social Universidade Federal da Paraíba - UFPB, e-mail: analayde@terra.com.br

*** Psicóloga e mestre em Psicologia Social pela Universidade Federal da Paraíba - UFPB e-mail: josevaniasco@gmail.com
} 


\begin{abstract}
The purpose of this study was to verify subjective well-being in mothers of children with an undefined diagnosis of soropositivity for HIV, assisted by the - SAE (Specialized Assistance Services) in the city of João Pessoa, Paraíba State. The convenience sample comprised 30 mothers of children with an undefined diagnosis of soropositivity, with ages varying from 19 to 39 years old (M=27, SD=5.18). The Subjective Well-Being Scale (SWBS) and a social and demographic questionnaire were applied. Data were processed by the SPSS (Statistical Package for the Social Sciences) statistic program and analyzed through bivariate statistics (student's t test; variance analysis). Results indicated instability in subjective well-being and association of the 'positive affect' factor with 'care time'. In conclusion, living a situation of undefined diagnosis of soropositivity for HIV/AIDS awakens various feelings related to the history of the epidemic, to coping with the problem and to the implications in different personal domains, among which maternity.
\end{abstract}

Keywords: Vertical transmission; HIV/AIDS; subjective well-being.

\title{
Resumen
}

El objetivo del estudio fue verificar el bienestar subjetivo de las madres de niños con diagnóstico de seropositividad al VHI indefinido, atendidas por un SAE (Servicio de Atención Especializada) en la ciudad de João Pessoa/ PB. La muestra de conveniencia se componía de 30 madres de niños con diagnóstico de seropositividad indefinido, con edades que variaban de los 19 a los 39 años $(M=27, D P=5,18)$. Se aplicó la Escala de Bienestar Subjetivo (EBES) y un Cuestionario Sociodemográfico. Los datos fueron procesados por el programa estadístico SPSS (Statistical Package for the Social Sciences) y analizados por medio de estadística bivariada (teste $t$ de Student; análisis de variancia). Los resultados mostraron inestabilidad en el bienestar subjetivo y asociación del factor Afectos Positivos con el Tiempo de Cuidado. (En conclusión,) Se concluye que la experiencia de una situación interrogativa ante el VHI/SIDA origina sentimientos diversos que tienen relación con la historia de la epidemia, el enfrentamiento y las implicaciones en los diversos dominios personales, entre ellos la maternidad.

Palabras claves: transmisión vertical; VIH/SIDA; bienestar subjetivo.

\section{Introdução}

0 atraso na saúde pública para a elaboração de estratégias de prevenção da AIDS em mulheres, entre as décadas de 1980 e 1990, esteve, de certo modo, relacionado à crença generalizada de que as mulheres estariam imunes à contaminação do vírus HIV (vírus da imunodeficiência humana) 
(Israel, 2002), ocasionando o que veio a ser chamado de "risco silencioso" (Barbosa \& Villela, 1996). No entanto, a partir da década de 1990, os setores de saúde pública do Brasil começaram a buscar maior compreensão do perfil da epidemia nas mulheres, já que, nessa mesma época, ocorreram as primeiras notificações de casos de AIDS (síndrome da imunodeficiência adquirida) em recém-nascidos (Israel, 2002). Sendo assim, o crescimento do número de casos pela transmissão vertical encontrou-se, em parte, relacionado ao aumento de casos da AIDS em mulheres em idade reprodutiva, consequência herdada do crescimento da transmissão heterossexual, que ficou conhecido como "heterossexualização" da doença e ocorreu partir da década de 1990 (Souza Jr, Szwarcwald, Barbosa Jr, Carvalho \& Castilho, 2004).

$\mathrm{Na}$ contemporaneidade, embora a notificação dos casos de AIDS seja maior entre os homens, a incidência da doença nessa população apresenta-se relativamente estável desde o ano 2000, o que não ocorre entre as mulheres, observando-se, com isso, o fenômeno da "feminização" da doença. Esse processo de "feminização" da AIDS associado à possibilidade de transmissão vertical do HIV vem demonstrando a necessidade de estratégias e políticas de saúde pública, uma vez que de $15 \%$ a $30 \%$ dos bebês nascidos de mulheres soropositivas contraem o vírus HIV (Neves \& Gir, 2006), quando se consideram os três aspectos de transmissão vertical, a saber: no pré-natal (transplacentar-intraútero); no perinatal, compreendendo aqui o momento do parto; e no pós-natal, por meio da amamentação. Com o avanço nos estudos sobre a AIDS, a terapia antirretroviral (TARV) passa a representar uma grande conquista no sentido de evitar a transmissão vertical. Segundo Paiva (2000), o tratamento com os antirretrovirais, o AZT (zidovudina), por exemplo, tem reduzido em $70 \%$ a infecção do HIV em crianças. A profilaxia com a terapia antirretroviral vem sendo bastante usada na prevenção da transmissão vertical, sendo aplicada tanto na mãe grávida no momento do pré-natal e parto quanto no recém-nascido, na vigilância após o parto. A resposta ao protocolo ACTG 076 decorrente de estudo comparativo realizado pelo AIDS Clinical Trial Group com dois grupos de mulheres grávidas e soropositivas assintomáticas, verificou que o grupo de mulheres que fizeram o uso de Zidovudina (AZT) durante a gestação, no momento do parto e nos seus bebês após o nascimento obteve um índice de transmissão do HIV de $8 \%$, índice bastante inferior quando comparado ao grupo de mulheres que fizeram uso de placebo e tiveram uma taxa de transmissão vertical de $25 \%$ (Connor et alli, 1994).

Assim, as diretrizes de prevenção do Ministério da Saúde, levando em conta o protocolo ACTG 076, sugerem algumas recomendações para profilaxia da 
transmissão materno-infantil do HIV, a saber: a) o oferecimento do teste anti-HIV para as gestantes, mediante aconselhamento, o que possibilitaria conhecer o status sorológico e, no caso de positividade do teste, uma maior adesão ao tratamento com o AZT durante o pré-natal, sendo o teste voluntário e confidencial; b) a quimioprofilaxia, que consiste em oferecer o AZT oral às gestantes soropositivas a partir da $14^{\mathrm{a}}$ semana de gestação, bem como administração do antirretroviral (ARV) injetável nas mães durante o trabalho de parto; c) a administração de AZT oral ao recém-nascido durante as primeiras oito horas após o nascimento e até a sexta semana de vida; d) a substituição do aleitamento materno pelo leite artificial ou leite humano pasteurizado disponível nos bancos de leite; 5) o acompanhamento das mães e da criança por meio de Serviços de Atendimento Especializado (SAE) até a definição do diagnóstico (Brasil, 2004).

O Ministério da Saúde implantou, a partir de 1994, ainda que de forma descentralizada, os Serviços de Assistência Especializada (SAE), ocorrendo a centralização dos serviços em 1997 por meio do repasse dos recursos às instituições de saúde (Silva, 2007). Considerando a crescente demanda assistencial e com base em projetos de serviços alternativos de atendimento à saúde, o Ministério da Saúde criou o Programa de Alternativas Assistenciais, com o objetivo de proporcionar, nos serviços da rede pública de saúde, centros de referência, estando o SAE no plano ambulatorial e, no plano de hospitalização, os hospitais-dia (HD) e a assistência domiciliar terapêutica (ADT) (Brasil, 2007). Segundo o Ministério da Saúde (2007), o SAE caracteriza-se por ser unidade assistencial de caráter ambulatorial, que promove ações de assistência, prevenção e tratamento às pessoas vivendo com HIV/ AIDS e aos familiares delas, contando com equipe de profissionais de diversas áreas da saúde, a saber: médicos, psicólogos, enfermeiros, farmacêuticos, nutricionistas, assistentes sociais, educadores, entre outros.

Não existe uma certeza com relação ao momento em que a criança contrai o HIV da mãe. Yoshimoto, Diniz e Vaz (2005) apontam que, segundo evidências clínico-laboratoriais, 70\% a 80\% das crianças infectadas pelo HIV só apresentam sintomas da doença após um longo tempo. Tal dado leva a crer que, possivelmente, a transmissão ocorra geralmente durante o parto. Assim, faz-se necessária o início precoce da terapia antirretroviral em mulheres HIV positivas que acontece durante todo o processo de pré-natal e não para no parto, estendendo-se por um tempo geralmente de dois anos, até ser realmente diagnosticada ou não a soropositividade ao HIV na criança. $\mathrm{O}$ não fechamento preciso do diagnóstico durante esse tempo torna essas crianças sorointerrogativas frente ao HIV. A incerteza com relação ao diagnóstico de sorologia só pode ser resolvida após os testes de HIV periódicos e a aplicação da terapia antirretroviral que perduram por dois anos, daí a expressão diagnóstico sorointerrogativo. 
Diante disso, observa-se que a AIDS traz grandes transformações para a vida dos envolvidos. Silveira e Carvalho (2002) afirmam que o desenvolvimento da doença altera o estilo de vida do indivíduo, o que, consequentemente, provoca reaçóes de adaptação tanto na família quanto no paciente. Da mesma forma, torna-se também difícil vivenciar uma gravidez e maternidade no contexto de soropositividade ao HIV, ainda mais quando se experimenta por aproximadamente dois anos a espera de um propenso diagnóstico para um filho.

A teoria do bem-estar subjetivo é usada no estudo para averiguar a forma como as pessoas se mostram satisfeitas consigo mesmas e com o meio em que vivem, bem como a maneira como equilibram as experiências conflituosas e felizes de suas vidas (Gusmão, 2004; Guedea, 2005). Nesse sentido, o bem-estar subjetivo pode ser entendido como a avaliação que as pessoas fazem de suas vidas, com relação a funcionamento e experiência psicológica adequadas, não significando que tal experiência seja a ausência de enfermidades ou de experiências desagradáveis, mas que há estado de satisfação consigo e com o ambiente no qual a pessoa está inserida (Ryan \& Deci, 2001). Assim, o bem-estar subjetivo é considerado um construto multidimensional por alguns pesquisadores (Ryan \& Deci, 2001), sugerindose que, na avaliação dele, sejam usadas diferentes medidas para avaliar esses três componentes (Gouveia et alii, 2003), como as medidas de autorrelato, embora Diener (2000) também faça referência a medidas fisiológicas.

As dimensões principais do bem-estar subjetivo compreendem um componente cognitivo e um componente afetivo (Diener, 2000), a saber: satisfação com a vida - componente cognitivo, que consiste em julgamento cognitivo de algum domínio específico na vida da pessoa; e os afetos positivo e negativo (componente afetivo), que se referem à frequência e à intensidade de emoções positivas e negativas experimentadas pela pessoa. Assim, se uma pessoa tem níveis elevados de bem-estar subjetivo, significa dizer que experimenta satisfação com a vida, frequentes emoções de contentamento e pouca frequência de emoções negativas, como tristeza ou raiva (Giacomoni, 2002). Por essas dimensōes, mediante complexa interação, perpassam as percepções acerca das experiências vividas, conforme o indivíduo faz o balanço das experiências prazíveis e desprazíveis, bem como julgamento cognitivo de algum domínio específico, comparando a situação atual com padrão subjetivamente determinado por ele (Albuquerque \& Troccóli, 2004; Schimmack, Radhakrishnan, Oishi \& Dzokoto, 2002).

O bem-estar subjetivo tem sido objeto de grande interesse de investigaçóes no meio acadêmico, observando-se os diversos estudos realizados nesse sentido (Diener, 2000; Llanes, 2001). Os estudos iniciais tinham como ênfase as condições objetivas relacionadas ao bem-estar subjetivo. Entretanto, verifica-se que não são apenas os indicadores objetivos que explicam o bem-estar das pessoas, mas a associação destes 
com os aspectos subjetivos da pessoa (Diener, Suh, Lucas \& Smith, 1999; Gouveia et alii, 2003). Dessa forma, conhecendo-se as condiçôes que promoveriam o bemestar, seria possível articular uma forma ampla do que seria vida satisfatória, bem como orientar as pessoas à vida saudável, à proporção que conhecessem tais condições (Giacomoni, 2002). Portanto, indicadores materiais influenciam na avaliação cognitiva que as pessoas fazem de suas vidas, e os aspectos sociodemográficos (renda, idade, gênero, situação conjugal, entre outros) estão relacionados com variações no bem-estar subjetivo (Koo, Rie \& Park, 2004). Nessa direção, existem estudos que investigam ou relacionam o efeito da doença ou da saúde com a qualidade de vida e o bem-estar subjetivo tanto de crianças como de adultos (Diogo, 2003; Assumpção Jr., Kuczynski, Sprovieri \& Aranha, 2000).

Mediante tais considerações, justifica-se importante o uso desse conceito no estudo do fenômeno da AIDS, uma vez que as repercussóes dessa moléstia trazem consigo mudanças adaptativas no estilo de vida de cada pessoa envolvida. Assim, objetivou-se, neste estudo, verificar o bem-estar subjetivo em mães de crianças com diagnóstico sorointerrogativo para o HIV, atendidas em um SAE na cidade de João Pessoa-PB.

\section{Método}

\section{Amostra}

Participaram deste estudo 30 mães (grávidas e não grávidas) de crianças que têm diagnóstico sorointerrogativo para o HIV/AIDS, isto é, mães soropositivas ao HIV que acompanham crianças (entre 0 e 2 anos) e que, portanto, têm um diagnóstico indefinido de soropositividade ao HIV. A faixa etária das crianças, citada anteriormente, justifica-se pelo fato de ser esse, geralmente, o tempo que se leva para a conclusão, após o respectivo tratamento com a terapia antirretroviral, de um diagnóstico de AIDS. As mães participantes tinham média de idade de 27 anos $(\mathrm{DP}=5,18)$, variando de 19 a 39 anos.

A pesquisa foi realizada no SAE (Serviço de Atendimento Especializado) Materno-Infantil de um hospital público da cidade de João Pessoa-PB. Esse serviço é considerado de referência na prevenção da transmissão vertical, bem como no tratamento e acompanhamento de mães e crianças soropositivas ao HIV/AIDS.

\section{Instrumentos}

a) Escala de bem-estar subjetivo (EBES), validada para o contexto brasileiro por Albuquerque e Trócolli (2004), é composta por 69 itens que buscam mensurar os três maiores componentes do bem-estar subjetivo, a saber: satisfação com a vida, afetos positivos e afetos negativos. Os itens do número 1 ao 47 dizem respeito 
aos afetos positivos e negativos, referindo-se a como os respondentes se sentiram ultimamente, e os itens do número 48 ao 62 buscam avaliar julgamentos relativos à satisfação ou insatisfação com a vida, ambos obedecendo uma escala tipo Likert, variando entre 1 (nem um pouco) a 5 (extremamente).

b) Questionário sociodemográfico: com o intuito de caracterizarem-se melhor as participantes, traçando-se, com isso, um perfil da amostra, foi procedido também um questionário sociodemográfico cujas questôes objetivavam a busca de informações sobre sexo, idade, grau de escolaridade, renda familiar, situação conjugal, nível de religiosidade e dados clínicos das participantes.

\section{Análise dos dados}

Os dados foram processados pelo programa estatístico SPSS (Statistical Package for the Social Sciences). Inicialmente foram procedidas análises descritivas (medidas de tendência central, distribuição de frequência) com o intuito de descrição das variáveis observadas. Posteriormente, foram feitos testes paramétricos (teste $t$ de Student, análise de variância-ANOVA), objetivando verificar as possíveis diferenças entre as médias dos grupos-critérios internos.

\section{Procedimentos}

Inicialmente foi feito um contato com a direção do hospital e do SAE para as devidas explicaçôes acerca dos objetivos do estudo e dos cuidados éticos adotados ao longo de todo o percurso metodológico. Em seguida, cada mãe foi também esclarecida e convidada a participar, sempre salvaguardando o livre-arbítrio de cada pessoa. Só após a autorizaçãoo expressa tanto da direção do hospital quanto da mãe respondente, bem como o consentimento do comitê de ética, os instrumentos puderam ser aplicados. É válido salientar que, antes da aplicação dos instrumentos, cada participante foi informada quanto aos propósitos do estudo e solicitada a assinar um termo de consentimento. Os instrumentos foram aplicados num ambiente sem interferências, em uma sala reservada, na qual permaneciam apenas o pesquisador e a mãe respondente.

\section{Resultados e discussão}

\section{Perfil sociodemográfico da amostra}

Mediante a análise dos dados, observou-se que a maioria das participantes era de mães jovens que se encontravam na faixa etária de 19 a 29 anos (73\%), grande parte das participantes (80\%) apresentou uma renda familiar que não ultrapassava a quantia de um salário mínimo, $73 \%$ das mães tinham união estável com seus parceiros e $70 \%$ não chegaram a concluir o ensino fundamental. 


\section{Tabela 1}

Características sociodemográficas das mães

\begin{tabular}{|c|c|c|c|c|}
\hline \multicolumn{2}{|c|}{ Variáveis sociodemográficas e clínicas } & \multirow{2}{*}{$\begin{array}{c}\begin{array}{c}\text { Frequência } \\
\text { (N) }\end{array} \\
12 \\
10 \\
3 \\
5\end{array}$} & \multirow{2}{*}{$\begin{array}{c}\begin{array}{c}\text { Percentual } \\
(\%)\end{array} \\
40 \\
33 \\
10 \\
17\end{array}$} & \multirow{2}{*}{$\begin{array}{c}\mathbf{p}^{*} \\
0,07\end{array}$} \\
\hline Faixa etária & $\begin{array}{l}19-24 \text { anos } \\
25-29 \text { anos } \\
30-34 \text { anos } \\
35-39 \text { anos }\end{array}$ & & & \\
\hline $\begin{array}{l}\text { Renda familiar } \\
\text { (em salário mínimo) }\end{array}$ & $\begin{array}{l}<1 \text { salário } \\
1 \text { salário } \\
2 \text { salários } \\
>2 \text { salários }\end{array}$ & $\begin{array}{c}12 \\
12 \\
3 \\
3\end{array}$ & $\begin{array}{l}40 \\
40 \\
10 \\
10\end{array}$ & 0,01 \\
\hline Estado civil & $\begin{array}{l}\text { Casada/convivente } \\
\text { Solteira }\end{array}$ & $\begin{array}{c}22 \\
8\end{array}$ & $\begin{array}{l}73 \\
27\end{array}$ & 0,01 \\
\hline Escolaridade & $\begin{array}{l}\text { Fundamental inc. } \\
\text { Fundamental com. } \\
\text { Médio inc. } \\
\text { Médio com. }\end{array}$ & $\begin{array}{c}21 \\
3 \\
2 \\
4\end{array}$ & $\begin{array}{c}70 \\
10 \\
7 \\
13\end{array}$ & 0,01 \\
\hline Religiosidade & $\begin{array}{l}\text { Nada religioso } \\
\text { Pouco religioso } \\
\text { Religioso } \\
\text { Muito religioso }\end{array}$ & $\begin{array}{c}1 \\
8 \\
6 \\
15\end{array}$ & $\begin{array}{c}3 \\
27 \\
20 \\
50\end{array}$ & 0,004 \\
\hline Número de filhos & $\begin{array}{l}1 \\
2 \\
3 \\
4 \\
5\end{array}$ & $\begin{array}{l}9 \\
9 \\
2 \\
7 \\
3\end{array}$ & $\begin{array}{c}30 \\
30 \\
7 \\
23 \\
10\end{array}$ & 0,11 \\
\hline Tempo de Cuidado & $\begin{array}{l}<6 \text { meses } \\
6 \text { meses }-1 \text { ano } \\
1-2 \text { anos }\end{array}$ & $\begin{array}{c}16 \\
6 \\
8\end{array}$ & $\begin{array}{l}53 \\
20 \\
27\end{array}$ & 0,06 \\
\hline
\end{tabular}

${ }^{*} \times 2-p \leq 0.05$

Os resultados apresentados no perfil sociodemográfico indicam uma maior prevalência do HIV/AIDS em mães com um menor grau de instrução e com baixas condições econômicas, o que confirma a "pauperização da epidemia". A literatura aponta que, no decurso histórico, alguns fatos ajudaram a, epidemiologicamente, entender melhor os impactos decorrentes do fenômeno da AIDS, entre eles está a "pauperização da epidemia". Sobre essa questão, Saldanha (2003) justifica que a propagação da epidemia de AIDS atinge os setores menos favorecidos da sociedade, uma vez que essa camada social apresenta-se mais exposta à marginalização, à falta de informação e acesso aos serviços de saúde, o que, consequentemente, dificulta açóes efetivas de prevenção. 
Apesar de a grande parte das participantes ter respondido ser mãe de mais de um filho, nenhuma delas respondeu ter algum filho soropositivo, bem como a experiência do "cuidar" de uma criança HIV positiva. Possivelmente tal dado justifique os resultados positivos do bem-estar subjetivo obtidos pela aplicação da EBES. A não vivência anterior do adoecimento de um filho por HIV/AIDS poderá ter sido um fator influente nos sentimentos positivos dessas mães perante a vida, o que, consequentemente, também interfere no bem-estar subjetivo, visto que este diz respeito à avaliação que cada pessoa faz sobre suas vivências, levando em conta os afetos positivos e negativos que se sente e a satisfação que se tem diante da autorrealização.

\section{Bem-estar subjetivo}

Conforme visto anteriormente, a escala de bem-estar subjetivo (EBES), cujo intuito principal consiste na avaliação que as pessoas fazem de suas vidas, é composta por três fatores: afetos positivos (AP), afetos negativos (AN) e satisfação com a vida (DP). Atendendo à recomendação de Diener, Suh, Lucas e Smith (1999) com relação a instrumentos de medida de bem-estar subjetivo (BES), o BES teve seus componentes avaliados separadamente neste estudo. Os escores nos fatores foram estimados pela média da soma dos pontos atribuídos a cada item que compõe cada um desses fatores e estão sintetizados na tabela 2 e vistos no gráfico 1.

Tabela 2

Escores de Avaliação do bem-estar subjetivo

\begin{tabular}{l|c|c|c}
\hline & Média & Desvio padrão & Amplitude \\
\hline Afetos positivos & 2,9 & 0,53 & $1,9-3,8$ \\
\hline Afetos negativos & 2,9 & 0,71 & $1,3-4,3$ \\
\hline Satisfação com a vida & 3,09 & 0,85 & $1,2-4,8$ \\
\hline
\end{tabular}

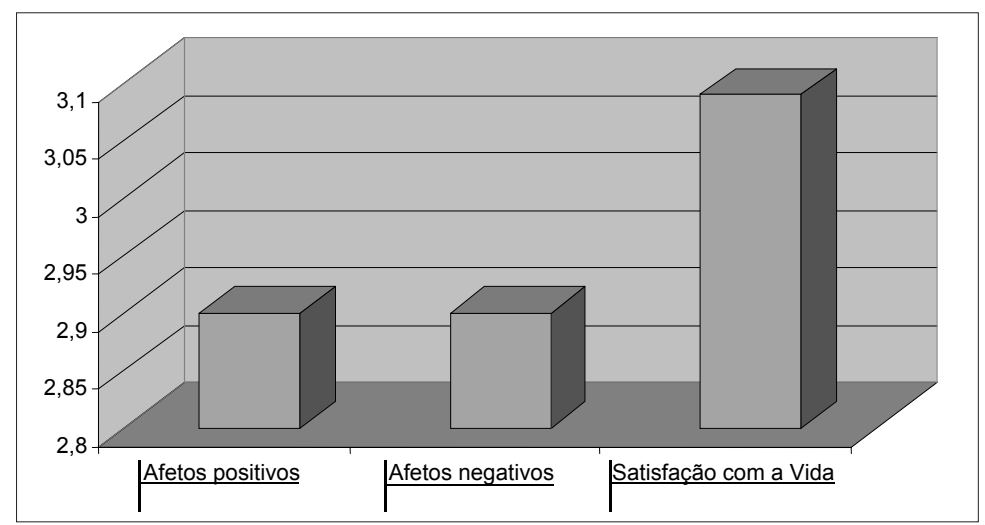

Gráfico 1: Avaliação do bem-estar subjetivo das mães 
Observa-se, nos fatores afetos positivos e afetos negativos, que as mães estudadas pontuaram abaixo do ponto de corte, nesse caso 3, enquanto que, no fator satisfação com vida, pontuaram um pouco acima. Apesar de terem apresentado uma satisfação com a vida, ultrapassando o limite do ponto de corte, isso não indica que estejam satisfeitas, uma vez que a amplitude das respostas nesse fator é grande $(1,2-4,8)$. No que diz respeito aos afetos, apesar das médias iguais, no fator afetos negativos, a amplitude de respostas também é grande. Isso indica que essas mães não se comportam de forma homogênea em suas respostas, existem respondentes que indicam estar bastante insatisfeitas com a vida, como também existem aquelas que demonstram ter bastantes afetos negativos. Nesse sentido, o que se observa pelos resultados obtidos é uma instabilidade no que diz respeito ao bem-estar subjetivo das mães em questão, possivelmente decorrente da vivência de uma situação de interrogação que emana sentimentos diversos, bem como dos próprios rumos atuais que o tratamento do HIV/AIDS tem tomado.

Em estudo com crianças portadoras do HIV/AIDS e os familiares delas, Pedro e Stobäus (2003) constataram sentimentos de culpa, incerteza, negação e desconfiança nas mães dessas crianças, os quais dificultavam o enfrentamento da situação. Moura e Praça (2006) apontam que, muitas vezes, as mulheres tomam conhecimento de sua condição de soropositividade no momento do pré-natal, parto ou mesmo no pósparto, fato que reforça os conflitos inerentes à maternidade no contexto da soropositividade ao HIV. Contudo, os avanços acerca do tratamento, o aumento da sobrevida e a melhora na qualidade de vida dos pacientes soropositivos têm mudado a forma de enfrentamento das pessoas perante a infecção. O sucesso com a terapia antirretroviral, por exemplo, ajudou a diminuir o medo da iminência da morte tão marcante no início da epidemia e justificou a necessidade de se construírem novas representações acerca da convivência com o HIV/AIDS. Carvalho, Morais, Koller e Piccinini (2007) apontam os avanços no diagnóstico e tratamento como um fator influente nas novas concepções da infecção HIV/AIDS, motivos pelos quais a AIDS têm se enquadrado como uma das doenças crônicas e tem-se intensificado a importância da otimização das relações sociais, laborais e afetivas de pessoas soropositivas, trazendo consigo aumento da qualidade de vida.

Nesse sentido, parece compreensível, no contexto do HIV/AIDS, a experiência de situaçõos que geram instabilidades emocionais que também afetam, de forma bastante expressiva, o bem-estar subjetivo das pessoas. 
Particularmente na maternidade soropositiva, essa questão se intensifica por tratar-se de uma pessoa que é tida como responsável pela vida de outra, cujos sentimentos que emanam dessa situação particular oscilam, podendo variar entre a dor e culpa como também responsabilidade e esperança. Se, por um lado, as mães do presente estudo passam por conflitos de aceitação, provavelmente oriundos do panorama histórico de medo e discriminação que sempre permeou a AIDS, ao mesmo tempo, diante do mérito nos avanços do tratamento, elas podem também se mostrarem fortes no enfretamento da situação. Em estudo com gestantes soropositivas para o HIV, Moura e Praça (2006) mostram que a ausência de sintomas e o uso de medicamentos reforçam a esperança na gravidez e, consequentemente, traz novas perspectivas de vida para mulheres que convivem com o HIV/AIDS.

Para comparar as médias dos fatores da BES com os dados sociodemográficos, faixa etária, estado civil, escolaridade, renda familiar, nível de religiosidade, além das variáveis, número de filhos e tempo de cuidado, foi usada a análise de variância (ANOVA). Em relação aos fatores afetos negativos e satisfação com a vida, não foi encontrada diferença estatisticamente significante para nenhuma das variáveis. Todavia, para o fator afetos positivos, observou-se a existência de uma associação com o tempo de cuidado (média tempo menor que 1 ano = 2,06; média tempo maior que 1 ano $=1,40 ; \mathrm{p}=0,03$ ), podendo-se, com isso, inferir que, quanto menor o tempo de cuidado, maior a emergência de afetos positivos.

A variável tempo de cuidado se refere ao tempo que a genitora presta cuidados a seu filho. No caso das gestantes, foi levado em consideração o período de gestação e, nas puérperas, considerou-se a idade da criança. Observa-se que quanto mais recente o tempo de cuidado mais intensos são os afetos positivos das participantes, indicando a urgência no enfrentamento do problema, proporcionando a essas mães vivenciar uma atmosfera de confiança e esperança que as conduzem numa expectativa positiva. Um fator a ser considerado nessa questão diz respeito ao tempo de tratamento da terapia antirretroviral e fechamento do diagnóstico em crianças, que podem levar até dois anos, originando momentos de ansiedade e cansaço que não são comuns no início do tratamento.

Foi usado ainda o teste $\mathrm{T}$ para analisar o poder discriminativo dos itens em relação aos grupos critérios internos que avaliam de forma positiva ou negativa os fatores afetos negativos, afetos positivos e satisfação com a vida, cujos itens que se mostraram significativos são apresentados na tabela 3 . 


\section{Tabela 3}

Itens dos fatores afetos negativos, afetos positivos e satisfação com a vida. Poder discriminativo em relação ao grupo de avaliação negativa e positiva (Test $\mathrm{t}$ )

\begin{tabular}{|c|c|c|c|c|}
\hline \multirow[t]{2}{*}{ Afetos negativos } & Grupos critério & Média & Desvio padrão & $\mathrm{p}^{*}$ \\
\hline & $\begin{array}{l}\text { Av- } \\
\text { Av+ }\end{array}$ & 3,69 & 0,94 & h 01 \\
\hline \multirow[t]{2}{*}{ Angustiada } & Av- & 4,23 & 0,92 & \\
\hline & $A v+$ & 2,76 & 1,09 & 0,001 \\
\hline \multirow[t]{2}{*}{ Apreensiva } & Av- & 3,92 & 1,38 & \\
\hline & $A v+$ & 2,41 & 1,27 & 0,005 \\
\hline \multirow[t]{2}{*}{ Receosa } & Av- & 3,85 & 0,89 & \\
\hline & $A v+$ & 2,18 & 1,01 & 0,03 \\
\hline Afetos positivos & Grupos critério & Média & Desvio padrão & $\mathrm{p}^{*}$ \\
\hline \multirow{2}{*}{ Ativa } & Av- & 2,73 & 0,88 & \\
\hline & Avt & 3,47 & 1,06 & 0,04 \\
\hline \multirow[t]{2}{*}{ Disposta } & Av- & 2,80 & 0,86 & \\
\hline & $A v+$ & 3,60 & 1,12 & 0,03 \\
\hline \multirow[t]{2}{*}{ Interessada } & Av- & 2,33 & 1,29 & \\
\hline & $A v+$ & 3,33 & 1,05 & 0,02 \\
\hline \multirow[t]{2}{*}{ Atenta } & Av- & 2,33 & 1,11 & \\
\hline & $A v+$ & 3,67 & 1,11 & 0,003 \\
\hline \multirow[t]{2}{*}{ Animada } & Av- & 2,20 & 1,32 & \\
\hline & $A v+$ & 3,40 & 1,06 & 0,01 \\
\hline \multirow{2}{*}{ Decidida } & Av- & 3,00 & 1,13 & \\
\hline & $A v+$ & 3,93 & 1,10 & 0,01 \\
\hline \multirow{2}{*}{ Engajada } & Av- & 2,67 & 1,17 & \\
\hline & $A v+$ & 3,53 & 0,83 & 0,02 \\
\hline \multirow[t]{2}{*}{ Entusiasmada } & $A v-$ & 2,40 & 0,91 & \\
\hline & $A v+$ & 3,60 & 0,99 & 0,002 \\
\hline Estimulada & $\begin{array}{l}\mathrm{Av}- \\
\mathrm{Av}+\end{array}$ & $\begin{array}{l}1,93 \\
3,27\end{array}$ & $\begin{array}{l}0,74 \\
1,39\end{array}$ & 0,003 \\
\hline Bem & $\begin{array}{l}A v- \\
A v+\end{array}$ & $\begin{array}{l}3,47 \\
3,47\end{array}$ & 1,12 & 0.01 \\
\hline Inspirada & $\begin{array}{l}A v- \\
A v+\end{array}$ & $\begin{array}{l}2,0 \\
3,20\end{array}$ & $\begin{array}{l}1,00 \\
1,01\end{array}$ & 0,03 \\
\hline Satisfação com a vida & Grupos critério & Média & Desvio padrão & $\mathrm{p}^{*}$ \\
\hline Estou satisfeito com minha vida & $\begin{array}{l}\mathrm{Av}- \\
\mathrm{Av}+\end{array}$ & $\begin{array}{l}1,70 \\
4,35\end{array}$ & $\begin{array}{l}0,82 \\
0,49\end{array}$ & 0.001 \\
\hline \multirow{2}{*}{$\begin{array}{l}\text { Tenho aproveitado as } \\
\text { oportunidades da vida }\end{array}$} & $A v-$ & 2,70 & 1,49 & \\
\hline & $A v+$ & 4,15 & 1,14 & 0,01 \\
\hline \multirow{3}{*}{$\begin{array}{l}\text { A minha vida está de acordo com o } \\
\text { que desejo para mim } \\
\text { Gosto da minha vida }\end{array}$} & Av- & 1,70 & 0,94 & \\
\hline & $A v+$ & 3,20 & 1,32 & 0,002 \\
\hline & $\begin{array}{l}A v- \\
A v+\end{array}$ & $\begin{array}{l}2,20 \\
4,40\end{array}$ & $\begin{array}{l}1,13 \\
0,68\end{array}$ & 0,001 \\
\hline Estou insatisfeito com minha vida & $\begin{array}{l}A v- \\
A v+\end{array}$ & $\begin{array}{l}3,80 \\
2,60\end{array}$ & $\begin{array}{l}1,32 \\
1,14\end{array}$ & 0,03 \\
\hline \multirow[t]{2}{*}{ Minha vida poderia estar melhor } & $A v-$ & 4,40 & 0,52 & \\
\hline & $A v+$ & 3,60 & 1,19 & 0,05 \\
\hline \multirow{2}{*}{$\begin{array}{l}\text { Tenho mais momentos de tristeza } \\
\text { do que de alegria na minha vida } \\
\text { Minha vida é "sem graça" }\end{array}$} & $\begin{array}{l}A v- \\
A v+\end{array}$ & $\begin{array}{l}3,90 \\
2,80\end{array}$ & $\begin{array}{l}0,88 \\
1,54\end{array}$ & 0,05 \\
\hline & $\begin{array}{l}\mathrm{Av}- \\
\mathrm{Av}+\end{array}$ & 3,90 & $\begin{array}{l}0,57 \\
1,09\end{array}$ & 0,001 \\
\hline \multirow{2}{*}{$\begin{array}{l}\text { Minhas condições de vida são } \\
\text { muito boas }\end{array}$} & Av- & 2,20 & 0,92 & \\
\hline & $A v+$ & 3,20 & 0,95 & 0,01 \\
\hline Considero-me uma pessoa feliz & $\begin{array}{l}A v- \\
A v+\end{array}$ & $\begin{array}{l}2,00 \\
4,30\end{array}$ & $\begin{array}{l}1,15 \\
0,86\end{array}$ & 0,001 \\
\hline
\end{tabular}

* significante a $\mathrm{p} \leq 0.05$ 
No que concerne aos afetos negativos, os itens aflita, angustiada, receosa $e$ apreensiva indicaram diferenças significativas no grupo que avaliou negativamente (Av-). Já nos afetos positivos, os itens que apontam uma atividade e disposição perante a vida (por exemplo, decidida, atenta, disposta, engajada, entusiasmada) apresentaram diferenças significativas no grupo que avaliou positivamente $(\mathrm{Av}+)$ esses afetos. E, finalmente, na satisfação com a vida, os itens que indicam uma valorização e cuidado com a vida (por exemplo, "Gosto da minha vida", "Estou satisfeito com minha vida", "Considero-me uma pessoa feliz") mostraram diferenças significativas no grupo que avaliou positivamente; enquanto que os itens "Estou insatisfeito com minha vida", "Minha vida poderia estar melhor", "Tenho mais momentos de tristeza do que de alegria na minha vida" $e$ "Minha vida é 'sem graça", que indicam insatisfação e tristeza perante a vida, apresentaram diferenças significativas no grupo que avaliou negativamente (Av-). Dessa forma, pôde-se concluir que, neste estudo, as variáveis que indicam um olhar de reprovação perante a vida foram avaliadas de forma negativa, enquanto que as variáveis que denotam afirmação, satisfação e aceitação da vida foram avaliadas de forma positiva pelas participantes.

Conforme mencionado anteriormente, essa realidade pode ser atribuída ao desfecho atual que a AIDS tem tomado, à ausência de uma experiência concreta anterior no que diz respeito à lida com um filho HIV+, bem como à própria ausência de sintomatologia da AIDS, uma vez que todas as participantes eram apenas soropositivas ao HIV. Seidl (2005) aponta o advento da terapia antirretroviral como um fator contribuinte para o surgimento de novos focos de estudos sobre a AIDS, entre eles o bem-estar subjetivo, o ajustamento e enfrentamento das pessoas que convivem com HIV/AIDS. No princípio da epidemia, os estudos identificavam que a carência de um tratamento eficaz e a presença dos sintomas colocavam as pessoas numa postura de enfrentamento esquivo e inativo, diferentemente, com os avanços da terapia antirretroviral, os estudos mais atuais se concentram na forma como as pessoas enfrentam o problema de forma positiva e produtiva (Seidl, 2005). Nesse sentido, os resultados aqui apresentados convergem para os propósitos de estudos contemporâneos da AIDS, haja vista que todas as participantes e seus filhos fazem uso da terapia antirretroviral, e grande parte se mostrou pessoas afirmantes da vida, o que as torna pessoas com um bem-estar subjetivo satisfatório. Contudo, visto que o enfrentamento é um aspecto que influencia diretamente a forma como essas mães avaliam suas vidas, a contemplação dessa dimensão torna-se uma limitação deste estudo. Portanto, sugerem-se estudos futuros que venham a suprir as possíveis relações entre o bem-estar subjetivo e enfrentamento dessas mães. 


\section{Considerações finais}

De um modo geral, verifica-se que a avaliação das mães acerca de suas vidas é influenciada por elementos sócio-históricos, psicológicos, emocionais e estruturais, o que evidencia a relação existente entre a história da epidemia, o posicionamento frente o adoecimento e vulnerabilidade, e as implicaçóes para os diversos domínios da vida da pessoa, entre eles a maternidade.

$\mathrm{Na}$ compreensão do fenômeno da AIDS e dentro dele a transmissão vertical, há que se considerarem elementos que abarcam não apenas aspectos clínicos ligados à afecção da doença, mas sim dimensões que vão além disso e encontram-se permeadas na vida das pessoas. Na relação existente entre AIDS e a questão do gênero, por exemplo, faz-se necessário levar em conta as dimensões macro e microssociais, analisando-as em termos políticos, econômicos e socioculturais: tais dimensões estão associadas ao tratamento desigual dado aos gêneros e, consequentemente, ao papel da mulher na família, na relação com seu parceiro sexual e nas crenças e valores da sociedade.

No tocante ao processo de "feminização" da AIDS, é preciso considerar que não é ser mulher que aumenta as possibilidades de vulnerabilidade ao HIV, uma vez que há diferença entre a vulnerabilidade de mulher jovem, pobre e com baixa escolaridade e a vulnerabilidade de mulher da mesma faixa etária, mas de condição econômica enormemente favorecida. Assim, sugere-se que as estratégias de saúde direcionadas para mulheres soropositivas e crianças expostas ao vírus HIV transcendam aos aspectos biológicos e abarquem também os valores pessoais e sociais do sujeito (discriminação, desigualdade social, entre outras), no sentido da responsabilidade social, buscando a atuação participativa da comunidade.

\section{Referências}

Albuquerque, A. S., \& Tróccoli, B. T. (2004). Desenvolvimento de uma escala de bem-estar subjetivo. Psicologia: teoria e pesquisa, 20 (2), 153-164.

Assumpção Jr., F. B., Kuczynski, E., Sprovieri, M. H. S. \& Aranha, E. M. G. (2000). Escala de avaliação de qualidade de vida: validade e confiabilidade de uma escala para qualidade de vida em crianças de 4 a 12 anos. Arquivos Brasileiros de Neuropsiquiatia, 58 (1), 119-127.

Barbosa \& Villela. (1996). A trajetória feminina da AIDS. In: Parker \& Galvão (org.). Quebrando o silêncio: mulheres e AIDS no Brasil. (pp. 17-32). Rio de Janeiro: Relume-Dumará. 
Brasil. Ministério da Saúde. Secretaria de Políticas de Saúde. (2004). Guia de tratamento: recomendaçôes para profilaxia da transmissão materno-infantil do HIV e terapia antirretroviral em gestantes. Brasília: Ministério da Saúde.

Brasil. Ministério da Saúde. (2007). PN-DST/AIDS. Serviço de Assistência Especializada (SAE) aos portadores de HIVIAIDS. Recuperado em 16 de agosto, 2007, de http://www.aids.gov.br/assistencia/link20.htm.

Carvalho, F. T, Morais, N. A, Koller, S. H \& Piccinini, C. A. (2007). Fatores de proteção relacionados à promoção de resiliência em pessoas que vivem com HIV/ AIDS. Cadernos de Saúde Pública, 23 (9), 2023-2033.

Connor, E. M., Sperling, R. S., Gelber, R., Kiselev, P., Scott, G., O Sullivan, M. J. et. al. (1994). Reduction of maternal infant transmission of human immunodeficiency virus type 1 with zidovudine treatment. New England Journal of Medecine, 331, 1173-1180.

Diener, E. (2000). Subjective well-being: the science of happiness and a proposal for a national index. American Psychologist, 55, 34-43.

Diener, E., Suh. E. M., Lucas, R. E., \& Smith, H. L. (1999). Subjective wellbeing. Three decades of progress. Psychological Bulletin, 125 (2), 276-302.

Diogo, M. J. E. (2003). Satisfação global com a vida e determinados domínios entre idosos com amputação de membros inferiores. Revista Panamericana Salud Publica, 13 (6), 395-399.

Giacomoni, C. H. (2002). Bem-estar subjetivo infantil: conceito de felicidade e construção de instrumentos para avaliação. Tese de doutorado não publicada. Curso de Pós-graduação em Psicologia do Desenvolvimento, Universidade Federal do Rio Grande do Sul, Porto Alegre.

Gouveia, V. V. et. al. (2003). A utilização do QSG-12 na população geral: estudo de sua validação de construto. Psicologia: teoria e pesquisa, 19 (3), 241 248.

Guedea, M. T. D. (2005). Modelo de estresse e bem-estar subjetivo em cuidadores de familiares idosos dependentes funcionais. Tese de doutorado não publicada. Instituto de Psicologia, Universidade de Brasília, Brasília.

Gusmão, E. E. S. (2004). A hipótese da congruência vocacional: consideraçôes acerca dos valores humanos e do bem-estar subjetivo. Dissertação de mestrado. Programa de Pósgraduação em Psicologia Social, Universidade Federal da Paraíba, João Pessoa. 
Israel, G. R. (2002). Como é que eu vou dizer para a gestante? Dilemas morais da oferta do teste anti-HIV a gestantes em trabalho de parto: uma contribuição da bioética. Dissertação de mestrado. Fundação Oswaldo Cruz, Escola Nacional de Saúde Pública, Rio de Janeiro.

Koo, J., Rie, J. \& Park, K. (2004). Age and gender differences in affect and subjective well-being. Geriatrics and Gerontology International, 4, 268-270.

Llanes, G. D. (2001). El bienestar subjetivo: actualidad y perspectivas. Revista Cubana Medicina General Integral, 17 (6), 572-579.

Moura, E. L. \& Praça, N. S. (2006). Transmissão vertical do HIV: expectativas e ações da gestante soropositiva. Revista Latino-Americana de Enfermagem, 14 (3), 405-413.

Neves, L. A. S. \& Gir, E. (2006). Crenças das mães soropositivas ao HIV acerca da transmissão vertical da doença. Revista Latino-Americana de Enfermagem, 14 (5), 140-147.

Paiva, M. S. (2000). Vivenciando a gravidez e experienciando a soropositividade para o HIV. Tese de doutorado não publicada, Escola de Enfermagem, Universidade de São Paulo, São Paulo.

Pedro, E. N. R \& Stobäus, C. D. (2003). Vivências e (com) vivências de crianças portadoras de HIV/AIDS e seus familiares: implicações educacionais. Revista Paulista de Enfermagem, 22 (1), 62-71.

Ryan, R. M. \& Deci, E. L. (2001). On happiness and human potentials: a review of research on hedonic and eudaimonic well-being. Annual Review of Psychology, 52, (1), 141-166.

Saldanha, A. A. W. (2003). Vulnerabilidade e construçôes de enfrentamento da soropositividade ao HIV por mulheres infectadas em relacionamento estável. Tese de doutorado não publicada. Universidade de São Paulo, Ribeirão Preto.

Schimmack, U., Radhakrishnan, P., Oishi, S. \& Dzokoto, V. (2002). Culture, personality, and subjective well-being: integrating process models of life satisfaction. Journal of Personality and Social Psychology, 82 (4), 582-593.

Seidl, E. M. F. (2005). Enfretamento, aspectos clínicos e sociodemográficos de pessoas vivendo com HIV/AIDS. Psicologia em Estudo, 10 (3), set-dez, 421-429. 
Silva, C. G. S. (2007). Serviço de assistência especializada (SAE): uma experiência profissional. Psicologia Ciência e Profissão, 27 (1), 156-163.

Silveira, E. A. A. \& Carvalho, A. M. P. (2002). Familiares de clientes acometidos pelo HIV/AIDS e o atendimento prestado em uma unidade ambulatorial. Revista Latino-Americana de Enfermagem 10 (6), 813-818.

Souza Jr., P. R. B, Szwarcwald, C. L, Barbosa Jr, A, Carvalho, M. F. \& Castilho, E. A. (2004). Infecção pelo HIV durante a gestação: estudo-sentinela parturiente, Brasil, 2002. Revista de Saúde Pública 38 (6): 764-72.

Yoshimoto, C. E, Diniz, E. M. A. \& Vaz, F. A. C. (2005). Evolução clínica e laboratorial de recém-nascidos de mães HIV positivas. Revista da Associação Médica Brasileira, 51 (2): 100-5. 